\title{
Conceptual Design of Super Environment-Conscious Intelligent Composites Incorporated with Life-Cycle Program of Self-Repair and Self-Collapse and Application for Environment-Conscious Interior Planning in Buildings
}

\author{
Toshio Fukushima
}

\author{
Department of Environmental Space Design, Faculty of Environmental Engineering, the University of Kitakyushu, \\ Kitakyushu 808-0135, Japan
}

Super environment-conscious intelligent composite materials incorporated with life-cycle program of self-repair during in service and selfcollapse after designed service life are proposed. One example is carbon short-cut fiber reinforced composites (CFRC) incorporated into matrix mortar with numerous two kinds of micro capsules imbedded with two smaller capsules containing nanometer-size reacting particle (calcium oxide $(\mathrm{CaO})$ or cement) and initiating one (highly water-containing resin particle). These double capsules are incorporated during mixing mortar and fiber as chemical admixture, but they are stably dispersed, not breaking during in service of composites. By triggering these double capsules by external electromagnetic wave having two different frequencies, these capsules are broken, and chemical reaction occurs. As the results, selfrepair is made to occur in the crack part during in service by the rather mild hydration reaction of cement, and self-collapse after designed service life by the rapid expansive hydration reaction of $\mathrm{CaO}$. These cement composites are intelligent due to the appropriate response to the controlled external electromagnetic trigger, and environment-friendly due to the elongation of service life by self-repair, and the easiness of demolition and recycling by self-collapse. The same concept is also applied to carbon continuous fiber reinforced plastic reinforced concrete (CFRPRC). Other than double microcapsules, glass optical fibers are also imbedded into matrix resin of FRP. By detecting the change of electric resistance of CFRP, we can predict the collapse. By triggering the embedded double capsules, self-repair and self-collapse are made to occur. These pseudobiological functions of self-repair and self-collapse are also incorporated into base concrete. These CFRPRC are enough to become intelligent and environment-friendly. By using well designed these super environment-conscious intelligent composites, I try to realize environmentconscious interior planning in which non-adhesive, but joint or set-in type interior construction are considered.

(Received June 20, 2005; Accepted August 18, 2005; Published December 15, 2005)

Keywords: conceptual design, environment-conscious intelligent composite, life-cycle program, self-repair/collapse, micro capsule, nanometer particle, carbon short-cut fiber reinforced cement composite, carbon continuous fiber reinforced plastic, electromagnetic wave, frequency control

\section{Introduction}

Recently, in the swell of the technological innovation that happens in the various technological and industrial fields, in a line by new energy, electronics, and the bio-technology, and so on, new materials shoulder the leading part of the high technology. Although the collapse of the bubble and longterm recessing of economy cast the shadow on the social vitality, and the serious global environmental problems common to the human race to be solved urgently force us to convert from the present European and American-style societies of mass production, mass consumption and mass waste into sustainable environment-conscious and resources circulation-oriented ones, the tidal wave that the technology of new materials play a guiding role to open a new epochmaking age. From the historical viewpoint, it can be said that the impact that the appearance and progress of new materials divide a new age in the human race society and the industrial form, playing the role of the "key technology" as a standardbearer of the technological innovation of that age, needless to cite he age classification by the material type of the Old Stone Age, the New Stone Age, the Bronze Age and the Iron Age, and the example of the Industrial Revolution by the mass production of good quality iron by the invention of the coke fireplace.

A building field was not an exception, but the innovation of the building materials gave the forms of the usual buildings and structures changes, and made possible to create the various new structures, and new residential spaces which have more advanced performances and functions. Building materials as supporting buildings were used in various styles so far to meet the demanded performances of buildings in that age and the climate. Various things are used in large quantities as building materials in the form which copes with demanded performances/functions in their use conditions, no matter what they are distinguished by the classification by the material character of organic, inorganic, metallic, and composite material, or by the use of structural and nonstructural material (or functional material), or by the production form of natural and artificial material. When the human race appeared on the earth, got out of the cave and set up for the first time a residence as a shelter from rain and the dew, avoiding the rage of the nature not necessarily mild to them, the residences were supported by such natural materials as wood, bamboo, grass, soil and stone and the use of these natural materials continued until at present. (There are many points to learn from them as the root forms of the ecomaterial in the meaning that they are easy to return to the ground because they are closely-intimate to the earth from the first, though there may be faults in the stable supply, the homogeneity of the quality, the strength, the durability, and so on.

With the progress of production engineering making possible the cheap and mass supply, metallic materials such as bronze, iron, aluminum and inorganic materials such as cement, concrete, glass, lime, and gypsum became to be used as building materials. In the first half of twentieth century, synthetic polymeric materials such as plastics and paints came on stage brilliantly, and became used as various functional building materials. Afterwards since about 1985, 
Table 1 Classification of building materials from the viewpoint of the usual material form.

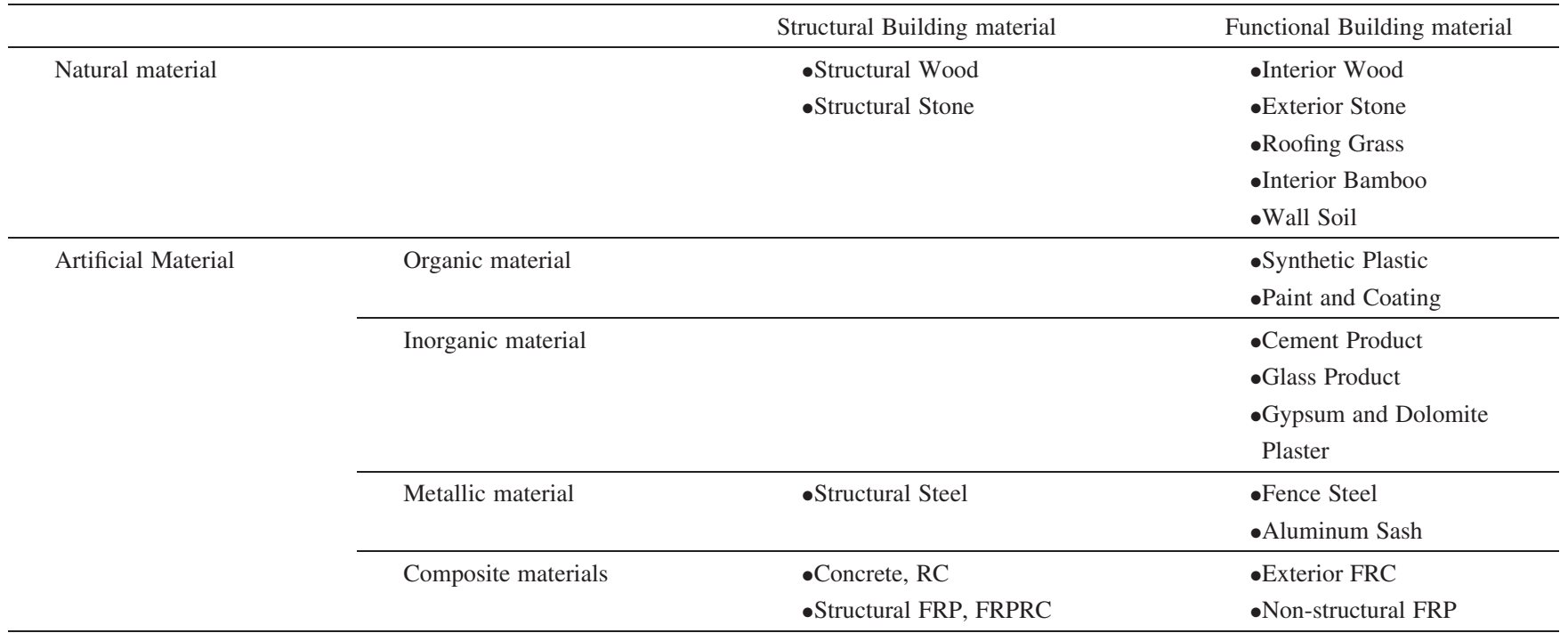

Table 2 Re-classification of building materials from the viewpoint of resources origin.

\begin{tabular}{|c|c|c|c|c|}
\hline & \multirow[b]{2}{*}{ Resources } & \multirow[b]{2}{*}{ Material } & \multicolumn{2}{|c|}{ Building Materials } \\
\hline & & & Structural materials & $\begin{array}{l}\text { Non-structural } \\
\text { materials }\end{array}$ \\
\hline Deriving from & Wood & Natural Wood, & Structural Wood & Interior Wood \\
\hline Renewable & & Natural Rubber & Earthquake-free Rubber & \\
\hline Reproductive & & Pulp & & Wall Paper \\
\hline \multirow{2}{*}{ Resources } & Grain & Starch & & Glue \\
\hline & Animal & Wool and Fur & & Insulation, Carpet \\
\hline Deriving from & Fossil & Synthetic Plastic & Structural FRP, FRPRC & Paints and Coatings \\
\hline Non-renewable & Ore & Steel & Structural Steel & Fence \\
\hline Non-reproductive & Rock & Glass & & Glass Wool \\
\hline \multirow[t]{2}{*}{ Resources } & & Cement & Concrete, RC & \\
\hline & Soil & Diatom Earth & & Soil Wall \\
\hline Deriving from & (Various & Gypsum + Paper & & Gypsum Board \\
\hline Both Resources & $\begin{array}{c}\text { types of } \\
\text { Composites) }\end{array}$ & Laminated Lumber & Structural Lumber & \\
\hline
\end{tabular}

in the rapid progress of materials science and technological innovation, with the appearance of new high performance fibers such as glass, carbon, aramid, fiber reinforced plastics (FRP), and fiber reinforced cement composites (FRC) fruited as advanced composite materials (ACM) which are the masterpieces of artificial materials, in a line of new metals, new polymers, and new ceramics, spread in various fields such as the universe, airplane, ship, railway, construction and buildings. However, in the present society seeking for sustainability that the importance of resources circulation and environment reservation are shouted strongly, these building materials have to be re-classified and evaluated from the viewpoint of their resources origin.

Table 1 shows the classification of the building materials from the viewpoint of the usual material form, and Table 2 shows the re-classification of the building materials from the viewpoint of resources origin.
This paper deals with the conceptual design of "super environment-conscious intelligent composite material" which merges and integrates the fundamental principles of "eco-material", "smart material", and "bio-material", improving the environmental harmony of fiber reinforced composites which took root as highly advanced building materials, based upon the analysis and consideration of the present conditions that about fifteen years has passed since the general idea of "the ecomaterial" was proposed.

\section{Historical Aspects of Composite Materials}

Composites, obtained from the combination of two or three different materials, are the most excellent masterpieces of artificial materials, because they have high strength/elasticity and high function surpassing the original component materials, compensating their defects and enlarging their merits. 
Concept of composite materials was known empirically from old age. Adobe bricks used in the Central East since ancient Egypt and Mesopotamia are kind of short-cut fiber reinforced clay reinforced with natural plant fibers. Earthen walls used in Japan used from old times are kind of double-reinforced clay reinforced both with short-cut fiber of natural straws, and with lattices of bamboo which is considered as natural continuous fiber reinforced composite. Many building materials are used as some composites, and very rarely as homogeneous single-phase materials. From microscopic point of view, even natural wood as one of the most important structural materials in housing is considered to be uniaxial cellulose fiber reinforced composites with lignine as binder. Artificial steel is also considered to be metallic solution composite reinforced with carbon with iron as binder.

Technology of composite materials, however, has drastically progressed in the latter half of the twentieth century through the development of materials science and the technological innovation with the development of advanced artificially fibers such as carbon, aramid, and glass, and bore fruit as advanced composites such as fiber reinforced plastics (FRP) and fiber reinforced cement composites (FRC). They are becoming more and more important in building and civil engineering fields. Due to their excellent material properties in-service such as lightweight, high strength and high corrosion-resistance, carbon, aramid, and glass continuous fiber reinforced plastics (CFRP, AFRP, and GFRP) are applied for non-metallic concrete reinforcements replacing steel liable to corrode in severe environmental conditions such as in the marine and seashore environment or in roads subjected to salt damage caused by deicer, and reinforcing fiber sheets for repair and aseismic reinforcement of deteriorated steel reinforced concrete (RC) structures. If we use continuous fiber reinforced concrete (FRPRC) by combining this lightweight, high strength, highly elastic and highly corrosion-resistant reinforcement with lightweight, high strength concrete, we can expect to improve material efficiency very much compared with ordinary RC by reducing the sectional areas and expanding the spans of columns and beams, and enlarging internal building space. Glass short-cut fiber reinforced unsaturated polyester resin (GFRUP) are used for kitchen units, bath tab, and water tank, etc. On the other hand, short-cut fiber reinforced cement composites (FRC) using new advanced fibers have very useful as lightweight, high strength and highly-durable exterior materials of, for example, new high performance external thermal insulation systems which are very useful from the viewpoint of the conservation of energy and resources.

As to these FRP, however, fibers are mostly bound with thermosetting resins, and are difficult to be recycled after designed service life. Only glass short-cut fiber reinforced unsaturated polyester can be reused as cement or sand replacing materials for concrete by pulverizing. As to these FRC, they are very difficult to be done material recycling except for reuse as they are as panel components, because short-cut fibers are mixed dispersively in them. We have to seek for the route to convert these high-tech composites into "ecomaterials" gentle both to the global environment and the

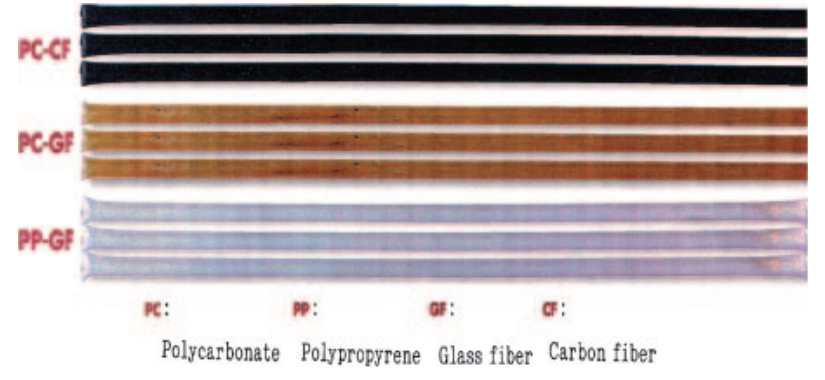

Fig. 1 Samples of recyclable carbon and glass continuous fiber reinforced thermoplastic reinforcement for construction.

dwelling environment and do environment-conscious materials design (ecomaterials design), considering the environmental harmony (ecobalance performance). ${ }^{1)}$

\section{Improvement of Ecobalance Performance of Ad- vanced Fiber Reinforced Composites}

Recently the concept of resources circulation and the environmental harmony have been appreciated as important in building field as well as other industrial fields, in order to establish sustainable society considering the balance among economy, the environment, and safety/long service life in the 21 st century. $^{2)}$ Effective use of environment-conscious materials (ecomaterials) as building materials, or the conversion of building materials into ecomaterials (ecomaterialization) are loudly cried for in these contexts.

We have tried to develop new non-metallic FRP reinforcement made of thermoplastic resins (FRTP: carbon and glass continuous fiber reinforced polycarbonate and polyvinyl chloride $^{3)}$ (Fig. 1). These FRTP's have high strength and high elasticity and show the complete elasticity during elongations that are the same with FRTS reinforcements, but they can be recycled 3 times by thermal warming. ${ }^{4)}$ On the other hand, for pitch-type carbon short-cut fiber reinforced cement composites (Pitch-CFRC), we examined experimentally how to convert FRC into ecomaterials by improving materials efficiency using ecocement (EC) (recycled cement obtained from urban wastes) and pulverized waste FRP powders (FRP) (glasses short-cut fiber reinforced unsaturated polyester resin) as replacing binder for ordinary Portland cement (OPC), and recycled fine aggregate (RS) as replacing for river sand $(\mathrm{S})$. We obtained fruitful results that material properties of these Pitch-CFRC's were comparable to or rather improved compared with ordinary Pitch-CFRC using only virgin resources, and that materials efficiency was improved very much. ${ }^{4)}$ Further, due to the finer particle size of EC powder, the flexural strength of Pitch-CFRC using EC is greater of that using OPC (Fig. 2). That gives the example of grade-up recycling in the sense of the improvement of performance by using recycled materials.

\section{Outline of Environment-Conscious Intelligent Com- posites}

The enlargement of various activities of the human beings on a global scale and accelerated mass consumption of 


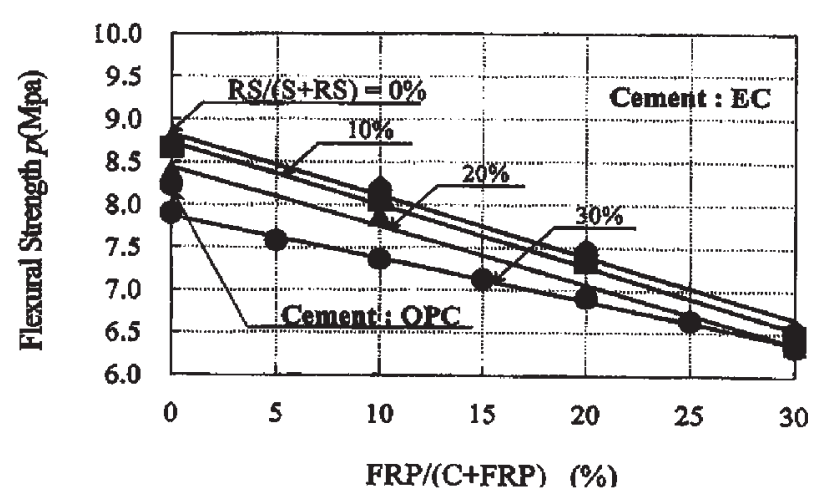

Fig. 2 Results of flexural strength of pitch-CFRC mixed with pulverized waste FRTS powders.

earthly resource in our age will inevitably leave our next generation with a state of devastated environment and of limited resource. In order to avoid our present circumstance getting worse, it is now imperative than ever to develop advanced materials with less consuming earthly resource and less polluting ecological environment. The futuristic material which is expected to be developed may be named as ecosmart composite structure (The history of mankind can be characterized from the material that they used at their age and we should call this the fifth generation material) as a successor to stone (the first generation material), bronze and iron (the second generation material), synthetics (the third generation material) and practical composites (the forth generation material). The fifth generation material is required to have such properties as (1) stronger and durable than existing materials, (2) elongated life expectancy, (3) ecologically compatible with environment. The fundamental aspect of the fifth generation material will be of added intelligent function of detecting own defect by itself and self-repairing and compatibility with the environment. In smart material, in order to perform self-monitoring and self-repairing, a sort of sensor, actuator and controller have to be equipped in its structure, which means that a kind of optical fiber, shape memory alloy, computer chip etc, are embedded in its matrix.

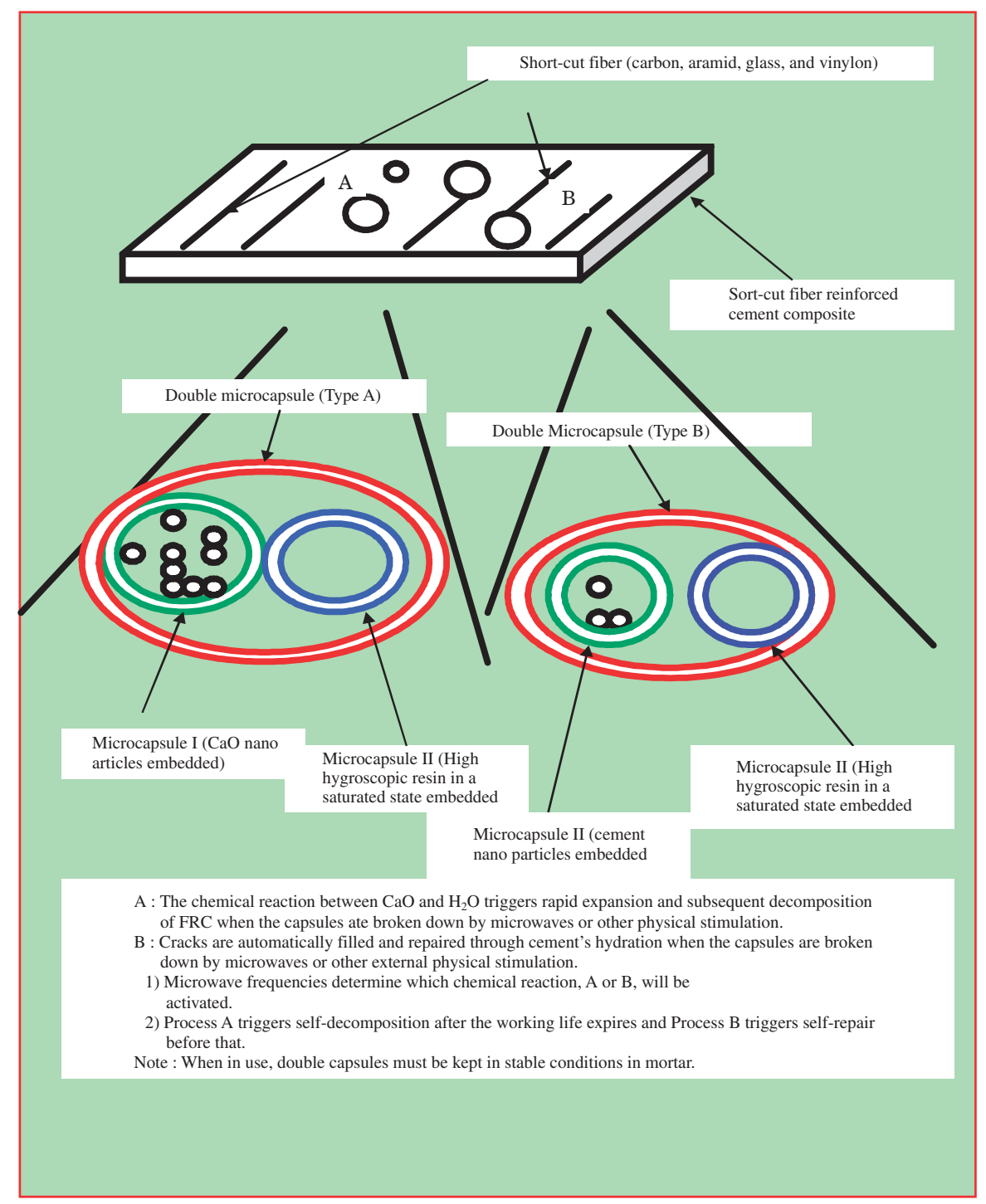

Fig. 3 Concept of super environment-conscious intelligent short-cut fiber reinforced cement composites with self-repairing/selfcollapsing functions. 


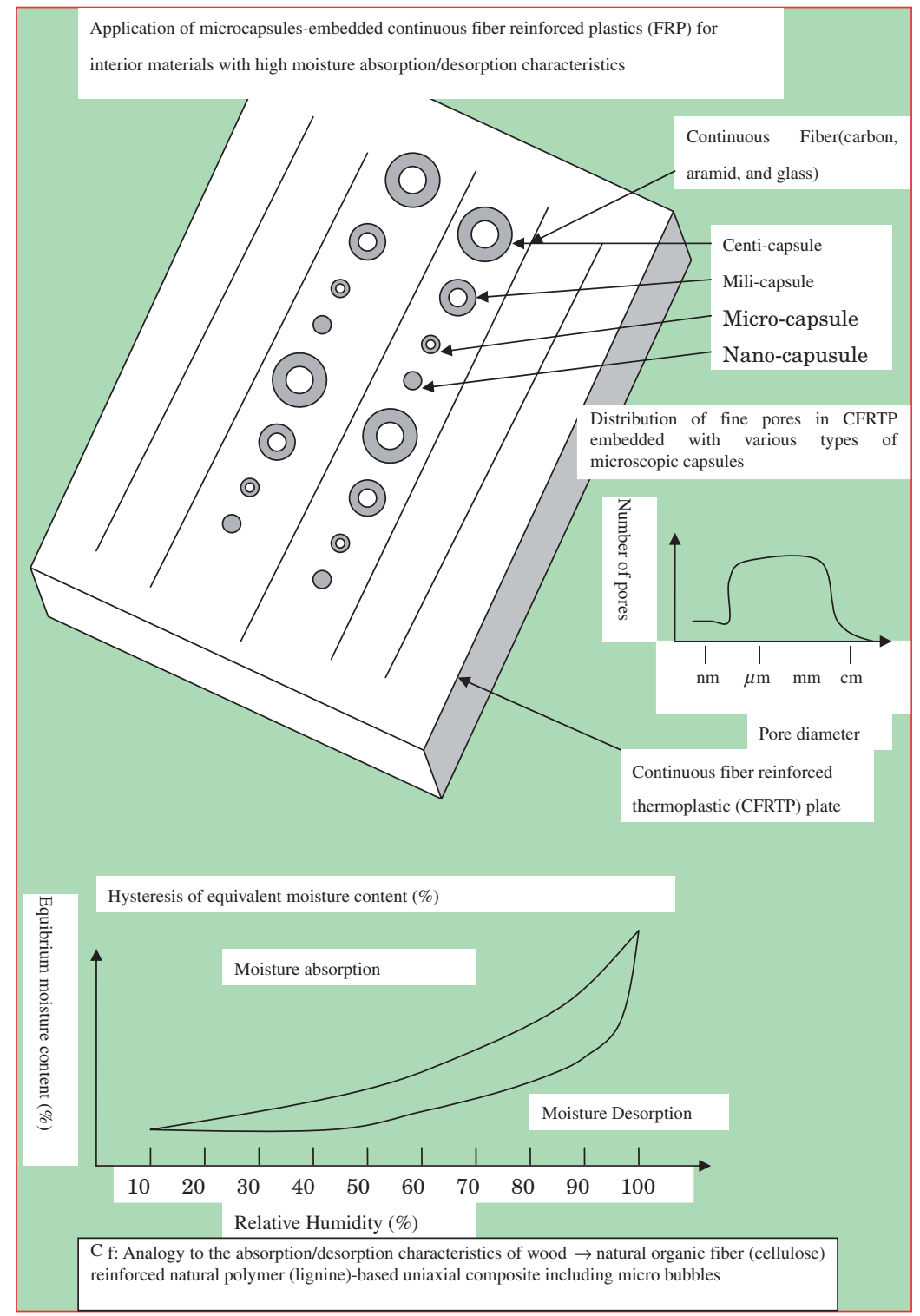

Fig. 4 Concept of $\mathrm{f}$ super environment-conscious intelligent continuous fiber reinforced thermoplastics (CFRTP) with self-repairing/selfcollapsing functions and high moisture controlling functions of absorption/desorption.

The complex combination of constituent element will cause the great burden to the environment, so that smart material is not so welcome to us in the future. Bio-degradable plastics or a single-phase material are less useful in the severe environment such as marine or cosmic space. In order to solve these problems, environment-conscious intelligent composites by the combination of microcapsule technology and fiber reinforced composites, which make possible both the required dynamic properties and ecobalance performance, was proposed. ${ }^{5)}$ Environment-conscious intelligent composites are not only lightweight, strong, and erosion-resistant but also smart and eco-friendly. They have the possibility of application in various fields, including buildings and civil engineering. They are, as it were, the coming ultimate materials in the future, integrating and surpassing the three most important materials in the present of smart materials, ecomaterials, and biomaterials.

\section{Super Environment-Conscious Intelligent Compo- sites}

\subsection{Conceptual design}

Super environment-conscious intelligent composite materials incorporated with life-cycle program of self-repair during in service and self-collapse after designed service life are proposed.6) One example is carbon short-cut fiber reinforced composites (CFRC) incorporated into matrix mortar with numerous two kinds of micro capsules imbedded with two smaller capsules containing nanometer-size reacting particle (calcium oxide $(\mathrm{CaO})$ or cement) and initiating one (highly water-containing resin particle) (Fig. 3). These double capsules are incorporated during mixing mortar and fiber as chemical admixture, but they are stably dispersed, not breaking during in service of composites. By triggering these double capsules by external electromagnetic wave having two different frequencies, these capsules are broken, and chemical reaction occurs. As the results, Self-repair is made 


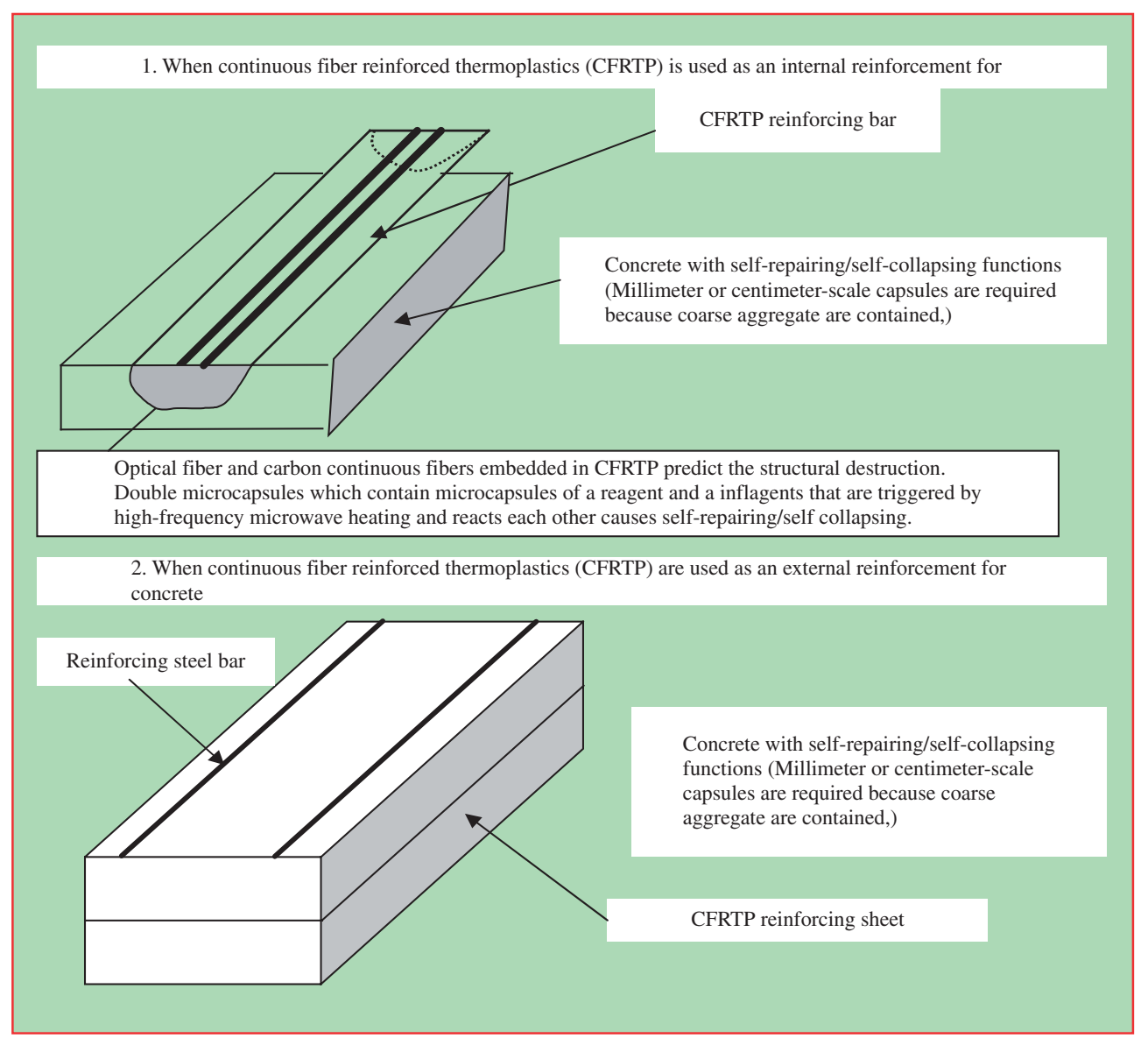

Fig. 5 Concept of super environment-conscious intelligent continuous fiber reinforced concrete with self-repairing/self-collapsing functions.

to occur in the crack part during in service by the rather mild hydration reaction of cement, and self-collapse after designed service life by the rapid expansive hydration reaction of $\mathrm{CaO}$. These short-cut fiber reinforced cement composites are intelligent due to the appropriate response to the controlled external electromagnetic trigger, and environment-friendly due to the elongation of service life by self-repair, and the easiness of demolition and recycling by self-collapse. They are possible to become super environment-conscious intelligent exterior materials, and applicable for high-function external thermal insulation systems in buildings contributing greatly to the reduction of life-cycle environmental load.

The same concept is also applied to carbon continuous fiber reinforced plastic reinforced concrete (CFRPRC). Other than double microcapsules, glass and optical fibers are also imbedded into matrix resin of FRP. By detecting the change of electric resistance or optical signal of CFRP (Fig. 4), we can predict the collapse. By triggering the embedded double capsules, self-repair and self-collapse are made to occur.

These pseudo-biological functions of self-repair and selfcollapse are also incorporated into base concrete. These CFRPRC are enough to become intelligent and environmentfriendly (Fig. 5).

\subsection{Technological aspects of breakthrough}

Although these super environment-conscious intelligent composite materials are attractive as he coming ultimate materials in the future, integrating and surpassing the three most important materials in the present of smart materials, ecomaterials, and biomaterials, there are some technological problems to be solved in the breakthrough of their development. The followings should be solved and technologically developed:

(1) Double microcapsules should be realized as the application of existing single microcapsule technology already prevailed in the fields of medical drugs and printing etc. These microcapsules are ingeniously incorporated, for example, as chemical admixture solutions in the matrixes of FRC and FRP and concrete in production, and have to be chemically stably dispersed, not breaking during in service of composites. If possible, nano-capsules, mili-capusles, centi-capsules are to be also developed, and by their effective combination, porous characteristics should be given for FRP in order to realize the pseudo-wooden absorption/desorption of moisture.

(2) Puedo-biolological genial information of self-repair and self-collapse should be also incorporated in the double microcapsules in order to effectively correspond to the external electromagnetic trigger.

(3) Self-repair and self-collapse should be skillfully controlled by external electromagnetic triggers having two different frequencies, for example, in far-infrared ray region. 


\section{Environment-Conscious Interior Planning by Mak- ing the Best Use of Ecomaterials and Non-Adhesive Construction Work}

Recently, the improvement of the dwelling environment has been emphasized, in proportion of the frequent occurrence of the problem of sick house syndrome by hazardous volatile organic compound (VOC), and the effective air condition system corresponding the global warming by the excessive energy consumption. So that rational resources circulation-oriented, and environment-conscious interior planning has become important. By using well designed these super environment-conscious intelligent composites, I try to realize environment-conscious interior planning in which non-adhesive, but joint or set-in type interior construction are considered. When super environment-conscious intelligent continuous fiber reinforced thermoplastics (CFRTP) with self-repairing/self-collapsing functions and high moisture controlling functions of absorption/desorption are used as interior materials and components by the nonadhesion type systems, the internal moisture environment will be greatly improved. On the other hand, by triggering by external microwave having two different frequencies, selfrepairing oculus in service during the designed service life, and self-collapse occurs after the designed service life. This makes possible the environment-conscious life-cycle design (eco-life-cycle design) in interior planning considering all thorough life-cycle.

\section{Conclusion}

Super environment-conscious intelligent composite materials incorporated with life-cycle program of self-repair during in service and self-collapse after designed service life were proposed.

(1) One example is carbon short-cut fiber reinforced composites (CFRC) incorporated into matrix mortar with numerous two kinds of micro capsules imbedded with two smaller capsules containing nanometer-size reacting particle (calcium oxide $(\mathrm{CaO})$ or cement) and initiating one (highly water-containing resin particle). These double capsules are incorporated during mixing mortar and fiber as chemical admixture, but they are stably dispersed, not breaking during in service of composites. By triggering these double capsules by external electromagnetic wave having two different frequencies, these capsules are broken, and chemical reaction occurs. As the results, self-repair is made to occur in the crack part during in service by the rather mild hydration reaction of cement, and self-collapse after designed service life by the rapid expansive hydration reaction of $\mathrm{CaO}$. These cement composites are intelligent due to the appropriate response to the controlled external electromagnetic trigger, and environment-friendly due to the elongation of service life by self-repair, and the easiness of demolition and recycling by self-collapse.

(2) The same concept was also applied to carbon contin- uous fiber reinforced plastic reinforced concrete (CFRPRC). Other than double micro capsules, glass optical fibers are also imbedded into matrix resin of FRP. By detecting the change of electric resistance of CFRP, we can predict the collapse. By triggering the embedded double capsules, self-repair and self-collapse are made to occur. These pseudo-biological functions of self-repair and self-collapse are also incorporated into base concrete. These CFRPRC are enough to become intelligent and environment-friendly.

(3) By using well designed these super environmentconscious intelligent composites, I tried to realize environment-conscious interior planning in which nonadhesive, but joint or set-in type interior construction are considered. When super environment-conscious intelligent continuous fiber reinforced thermoplastics (CFRTP) with self-repairing/self-collapsing functions and high moisture controlling functions of absorption/ desorption are used as interior materials and components by the non-adhesion type systems, the internal moisture environment will be greatly improved. On the other hand, by triggering by external microwave having two different frequencies, self-repairing occurs in service during the designed service life, and selfcollapse occurs after the designed service life. This makes possible the environment-conscious life-cycle design (eco-life-cycle design) in interior planning considering all thorough life-cycle.

\section{Acknowledgements}

The author would like to express heartful thanks to the New Energy and Development Organization of Japan (NEDO) for the kind permission to the official publishing in English version of the part of the Reports of Leading Research of New Energy and Development Organization of Japan (1998, 1999). Thanks are expressed to the Japan Chemical Innovation Institute for kind help for summing up these reports.

\section{REFERENCES}

1) T. Fukushima: Mater. Sci. Forums 426-432 (2003) 3323-3328.

2) T. Fukushima: Environment-conscious Materials and Systems for Sustainable Development (Proc. RILEM International Symposium, held on Sep. 6-7, 2004, at Koriyama, Japan, 2004) pp. 277-285.

3) T. Fukushima, K. Sakayama and S. Hashimoto: Proc. 5th Japan Interntl. SAMPE Sym. on Challenging Materials and Processing Technology Aiming for New Industrial Application, (Tokyo, Japan, 1997) pp. 14571462

4) T. Fukushima, K. Yanagi, T. Sone and H. Kurauchi: Proc. 2001 2nd Interntl Conf. on Engineering Materials, (held on August 16-21 at San Jose, U.S.A., 2001) pp. 53-66.

5) Report of Leading Research of New Energy and Development Organization of Japan (1998) (NEDO-PR-9910) Survey Research on Advanced Materials Technology by Microcapsules (in Japanese).

6) Report of Leading Research of New Energy and Development Organization of Japan (1999) (NEDO-IT-0029) Survey Research on Advanced Materials Technology by Microcapsules (in Japanese). 